\title{
Our Enigmatic Sun
}

\author{
Eric Priest ${ }^{1}$ \\ ${ }^{1}$ Department of Mathematics, University of St Andrews, ST ANDREWS KY16 9SS, UK \\ email: eric@mcs.st-and.ac.uk
}

\section{Introduction}

There are many reasons to study the Sun: it is of great scientific interest in its own right; it has profound influences on the Earth; and it is of crucial importance for astronomy, since it is the only star where we can observe fundamental cosmic processes in great detail. However, many of the basic properties of the Sun are still a mystery and so the Sun is one of the liveliest branches of astronomy to which many students are currently being attracted.

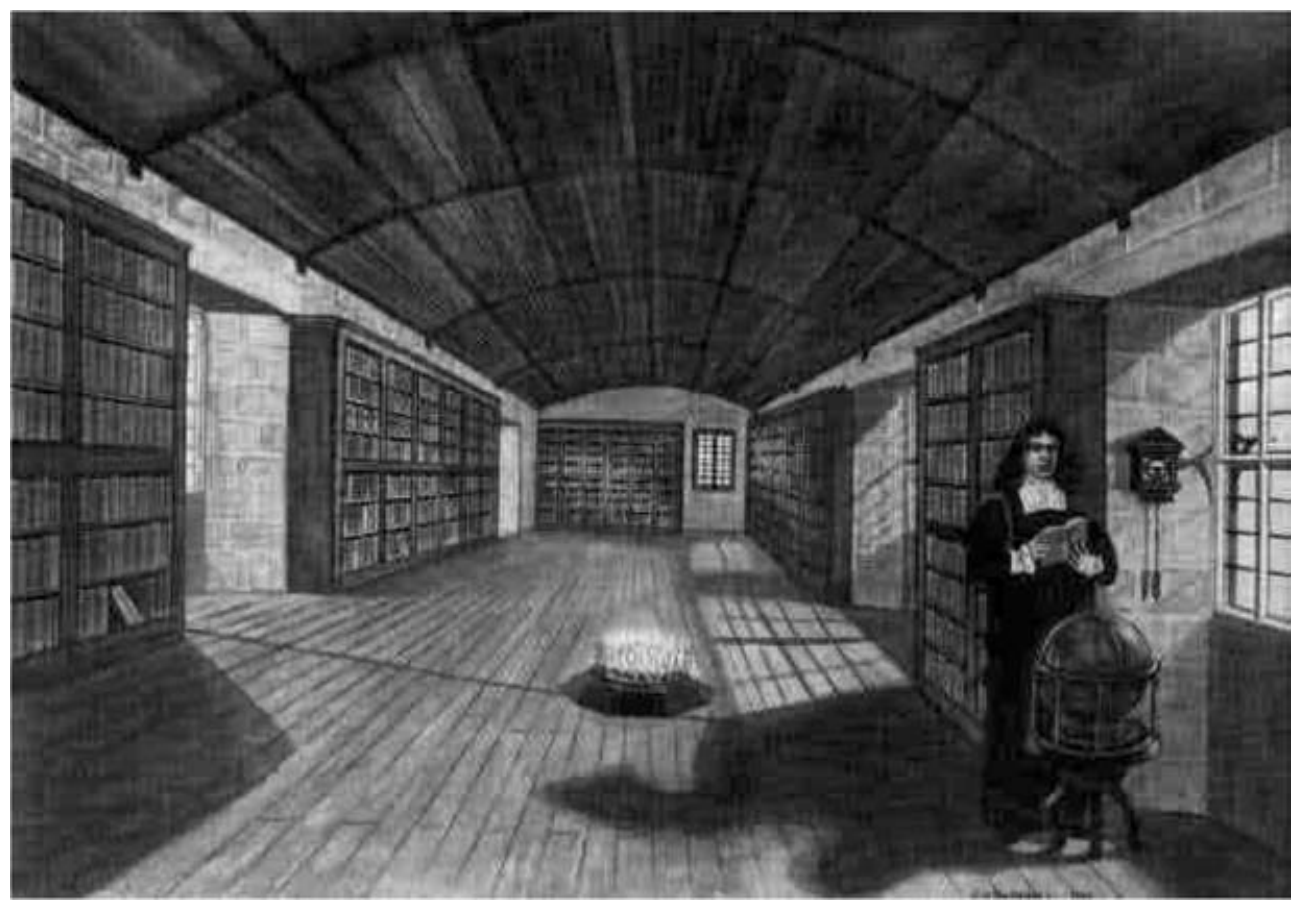

Figure 1. High-resolution image of photosphere (G. Scharmer, Swedish Academy of Sciences

Traditionally, there has been a very close link between mathematics and astronomy. For example, James Gregory invented the reflecting telescope but was also a co-founder with Newton and Leibniz of Calculus. He was appointed the first regius professor of mathematics at St Andrews at the age of 30 in 1668, and was give upper parliament hall as his laboratory (Figure 1), where you can see to this day the meridian line on the floor, along which he aligned his telescope. He left St Andrews in 1674 because of "a prejudice the masters of the University did take at the mathematics", and went to Edinburgh, but tragically he died a year later - at the age of 37 . He discovered many techniques 


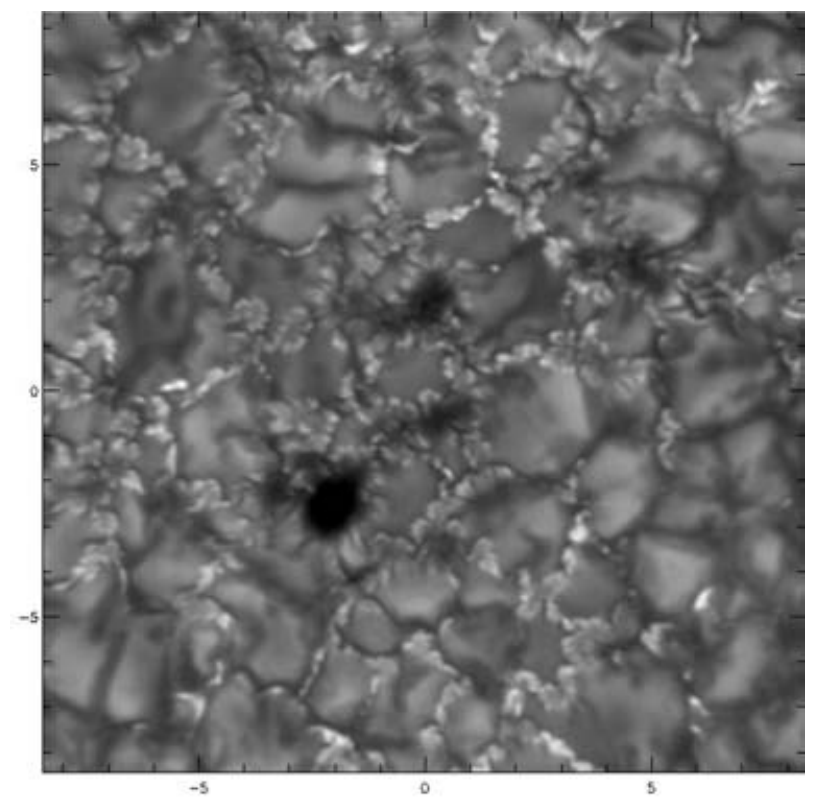

Figure 2. High-resolution image of photosphere (G. Scharmer, Swedish Academy of Sciences

of calculus such as the general binomial theorem, Taylor expansions, the ratio test for convergence of a series and how to change the variable in integration.

My theme today is that our Sun (which is half-way through its life expectancy of 10 billion years) is full of surprises and that many of them are caused by the magnetic field. The Sun, being a plasma in the fourth state of matter, behaves quite differently from a normal gas, since a magnetic field and a plasma are coupled in an intimate, subtle way.

\section{Overall Structure of the Sun}

The interior of the Sun consists of a core where the energy is generated, and the outer $30 \%$ is a turbulent connection zone. The atmosphere has three parts, namely the photosphere at a temperature of about $5780 \mathrm{~K}$ and above it the atmosphere and corona. The latest models of the photosphere and corona (Carlsson and Stein, 2002) show them to be highly time-dependent and inhomogenous. The photosphere is covered with turbulent cells - granulation with a size of about $1 \mathrm{Mm}$ and supergranulation on a scale of $15 \mathrm{Mm}$. The latest amazing images from the Swedish solar telescope of G. Scharmer and colleagues (Figure 2), described at this conference by Matts Carlsson, reveal bright points, flowers and ribbons at the edges of individual granules where the magnetic field is concentrated.

Sunspots are dark because they are cool (at about $3700 K$ ), because the magnetic field stops granulation and are basically locations where huge magnetic tubes are vertical to the solar surface. The penumbral striations around the central dark umbra are due to the spreading of the magnetic field from the vertical, but more stunning images from the Swedish telescope have revealed many strange and unexplained features of the penumbra (Scharmer et al, 2002; van der Voort et al, 2004). Some of these features are explained in a new model described at this symposium by Jack Thomas (Figure 3), in which the dark filaments are low-lying and held down by flux pumping of surrounding granules and the bright filaments are inclined at a smaller larger angle to the solar surface and lie along magnetic field lines that extend much further from the spot. 


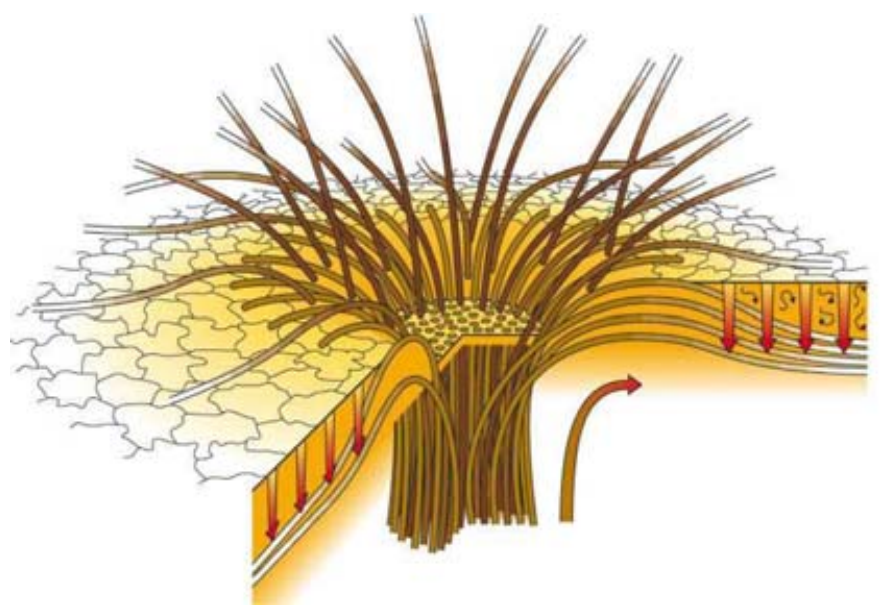

Figure 3. New model of sunspot penumbra due to Thomas et al, 2002 and Weiss et al, 2004

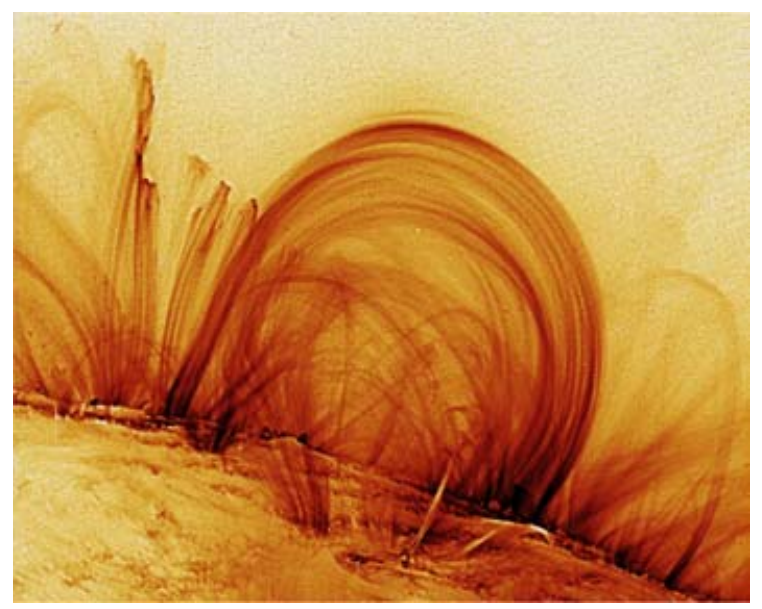

Figure 4. Image of the corona from TRACE (Courtesy A. Title)

The corona may be observed both at solar eclipses, when the glare of the photosphere is cut out by the moon or by a disc in a coronagraph, and also direct by soft $x$-ray or EUV telescopes. The magnetic field dominates coronal plasma, both heating and creating the beautiful observed structures. However, understanding the details of how the magnetic field heats the corona to a million degrees or more remains a major puzzle. Telescopes such as the Soft X-ray Telescope on Yohkoh (launched in 1991) or TRACE (launched in 1998) have revealed the corona to be a magnetic world with an amazingly rich variety of MHD phenomena. For example, images such as Figure 4 show highly intricate structure aligned along the magnetic field lines. This is caused by a combination of the dominance of the magnetic field by the plasma, the small size of the heating source and the small thermal conduction across the magnetic field.

\section{Magnetohydrodynamics}

The equations of magnetohydrodynamics (MHD) may be used to model the interaction of a magnetic field with a plasma, treated as a continuous medium. They are a unification of the equations of fluid mechanics and of Maxwell and are a set of partial differential 
equations for the behaviour in space and time of the magnetic field $(\mathbf{B})$, plasma velocity $(\mathbf{v})$, density $(\rho)$, pressure $(p)$ and temperature $(T)$. They include equations of induction, continuity and motion, namely

$$
\begin{gathered}
\frac{\partial \mathbf{B}}{\partial t}=\nabla \times(\mathbf{v} \times \mathbf{B})+\eta \nabla^{2} \mathbf{B} \\
\frac{d \rho}{d t}=-\rho \nabla \cdot \mathbf{v} \\
\rho \frac{d \mathbf{v}}{d t}=-\nabla p+\mathbf{j} \times \mathbf{B}+\rho \mathbf{g}
\end{gathered}
$$

where

$$
\nabla \cdot \mathbf{B}=0 \quad \text { and } \quad p=R \rho T,
$$

together with an energy equation and an equation

$$
\mathbf{j}=\nabla \times \mathbf{B} / \mu
$$

for the electric current $(\mathbf{j})$. The equations are nonlinear and to me as beautiful as the Sun. They are impossible to solve completely, and so we approximate them in imaginative ways and use them to construct models for many complex phenomena on the Sun.

The induction equation (1) describes how the magnetic field at a fixed point in space changes in time due to two terms on the right-hand side, namely, transport of the magnetic field with the plasma and diffusion of the magnetic field through the plasma. The first term on the right is very much larger than the second in most of the Universe and so the magnetic field is frozen to the plasma and hangs on to its energy.

The exception is in singularities where the gradient of the magnetic field, and therefore by (4) the electric current, is extremely large. These singularities form at null points where the magnetic field vanishes and the magnetic field lines break and reconnect. In the process, the magnetic energy is converted into other forms: in particular, the large electric currents imply strong ohmic heating $\left(j^{2} / \sigma\right)$. Such magnetic reconnection is thought to operate at the core of a solar flare and in myriads of coronal heating events.

\section{Three-Dimensional Reconnection}

The theory for magnetic reconnection in two dimensions is well understood, but recently we have started trying to understand how it works in three dimensions, where there are many new features. First of all, the structure of a null point is quite different. In two dimensions, you find either $X$-type null points or 0 -type nulls, but in three diemsnions there are two families of field lines through the null, namely, an isolated spine field line, which approaches (or recedes from) the null from two sides, and a fan surface of field lines which recede from (or approach) the null, as shown in Figure 5.

In two dimensions, separatrix curves coming from $X$-points split the plane into topologically distinct regions. In three dimensions, on the other hand, separatrix surfaces split the volume into topologically distinct regions, in the sense that all the field lines in one region have the same sources as their start-points and end-points. The separatrix surfaces intersect in special field lines called separators, which join one 3D null point to another.

Three-dimensional reconnection can occur either at a null point or in the absence of a null point (e.g., Schindler, Hesse \& Birn, 1988; Priest and Forbes, 2000). At a null, spine reconnection has the current concentrate along the spine, whereas fan reconnection has it 


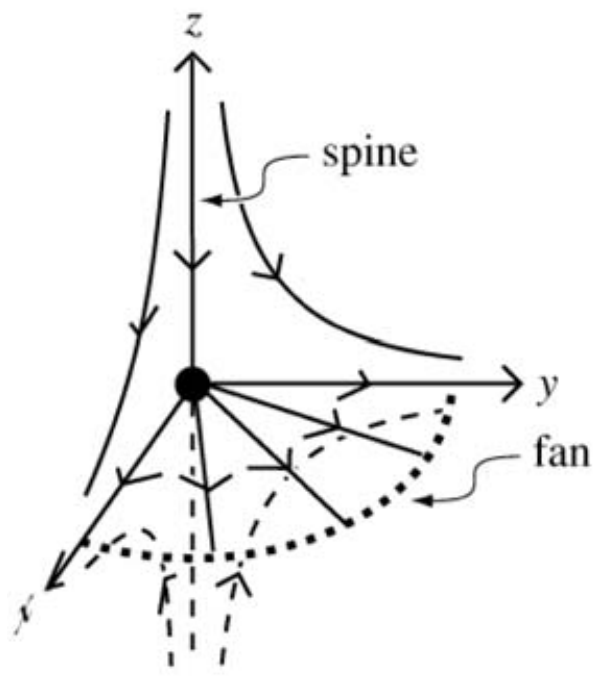

Figure 5. A three-dimensional null point

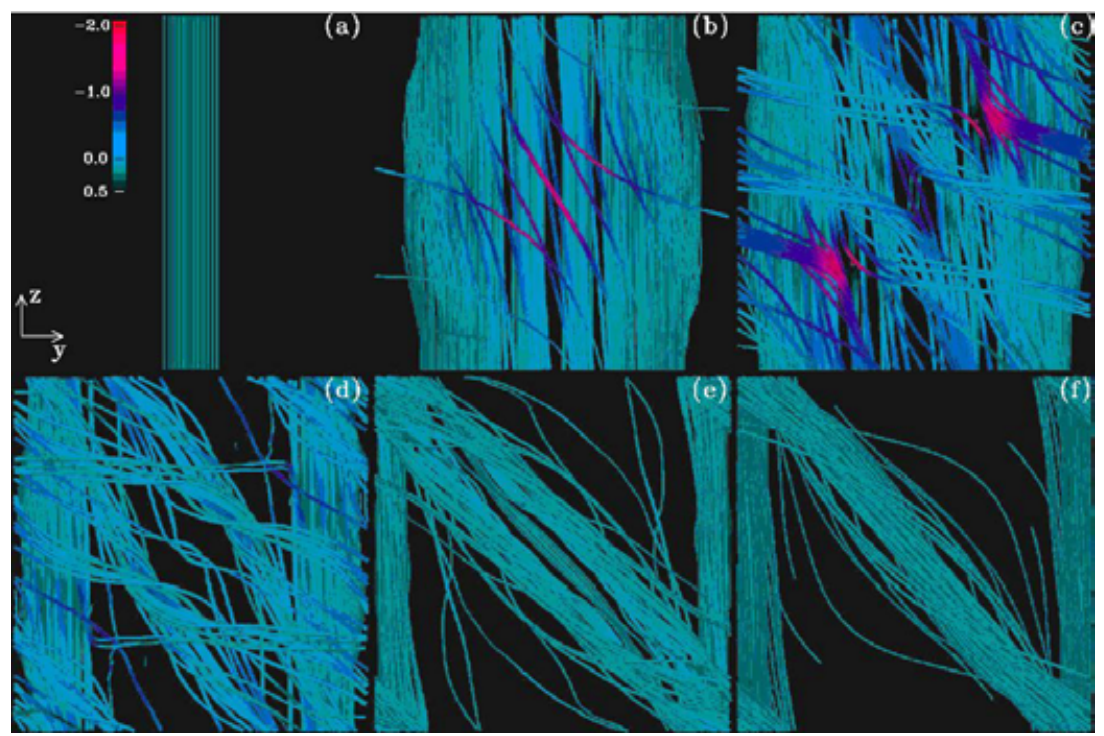

Figure 6. Field lines reconnecting as two flux tubes approach (Linton and Priest, 2003)

concentrate along the fan, and separator reconnection along the separator. An example of non-null reconnection has been studied by Linton and Priest, in which they started with two flux tubes at right angles, forced towards each other by an initial stagnationpoint flow. The field lines for one tube during the reconnection process reveal several features (Figure 6). The reconnection fragments and occurs in several places. Complex twisting and braiding is produced, with the conservation of magnetic helicity giving a final flux tube having a twist $(\Phi)$ of $\pi$ : thus, equating the initial mutual helicity and final self-helicity implies that

$$
F^{2}=2 \times \frac{\Phi}{2 \pi} F^{2}
$$




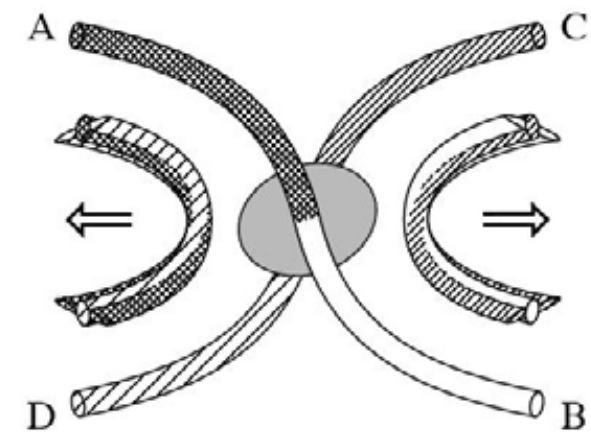

Figure 7. Breaking and partial rejoining of two flux tubes in $3 \mathrm{D}$ to form four new flux tube

giving $\Phi=\pi$. Furthermore, the higher the magnetic Reynolds number, the more reconnection locations and the more braiding is produced.

Another difference between $2 \mathrm{D}$ and $3 \mathrm{D}$ concerns the nature of field line velocities $(\mathbf{w})$ obeying

$$
\mathbf{E}+\mathbf{w} \times \mathbf{B}=\mathbf{0},
$$

so that outside a diffusion region, $\mathbf{w}$ and the plasma velocity $(\mathbf{v})$ are equal. In $2 \mathrm{D}$, inside the diffusion region, w does not exist for an isolated region (Priest, Hornig and Pontin, 2003) i.e., there is no solution for $\mathbf{w}$ to Equation (5). The field lines continually change their connections in the diffusion region, while flux tubes split, flip and in general do not rejoin perfectly (Hornig and Priest, 2003; Pontin, Priest and Hornig, 2003), as indicated in Figure 7.

\section{SOHO (The Solar and Heliospheric Observatory)}

SOHO was launched in 1995 and is orbiting the Sun in phase with the Earth. It is observing the Sun continuously for the first time and is giving the first comprehensive view of the Sun. Indeed, it has produced many surprises which are revolutionising our understanding of the Sun. I can only touch on a couple of questions here.

\subsection{Flares and Coronal Mass Ejections}

One of the instruments, LASCO, has coronagraphs which produce an artificial eclipse and have discovered huge ejections of plasma and magnetic flux called coronal mass ejections, which can reach the Earth and affect communications and space satellites. On October 28, 2003, an unusually large and complex active region was crossing the Sun. It spawned the third largest solar flare ever recorded and produced a coronal mass ejection which reached the Earth in a record 19 hours. This in turn triggered wonderful displays of aurora in St Andrews, which many of us watched with glee. A week later, the fireworks continued and the biggest flare ever recorded took place.

The overall picture of an eruptive flare is that a twisted sheared magnetic arcade stores magnetic energy in excess of potential and loses equilibrium or goes unstable and begins to erupt. The eruption itself drives reconnection underneath the erupting flux tube and produces a rising arcade of loops, which are powered by reconnection in an overlying current sheet. Observations from the TRACE satellite have imaged the current sheet and simultaneous RHESSI observations (Figure 8) have shown contours of hard $x$-rays, 


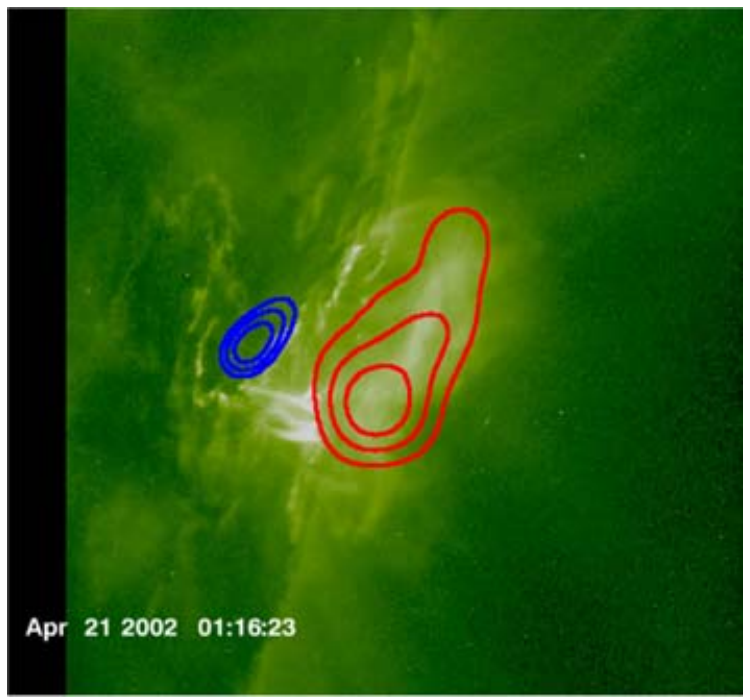

Figure 8. A solar flare observed by TRACE and RHESSI (Fletcher, 2004)

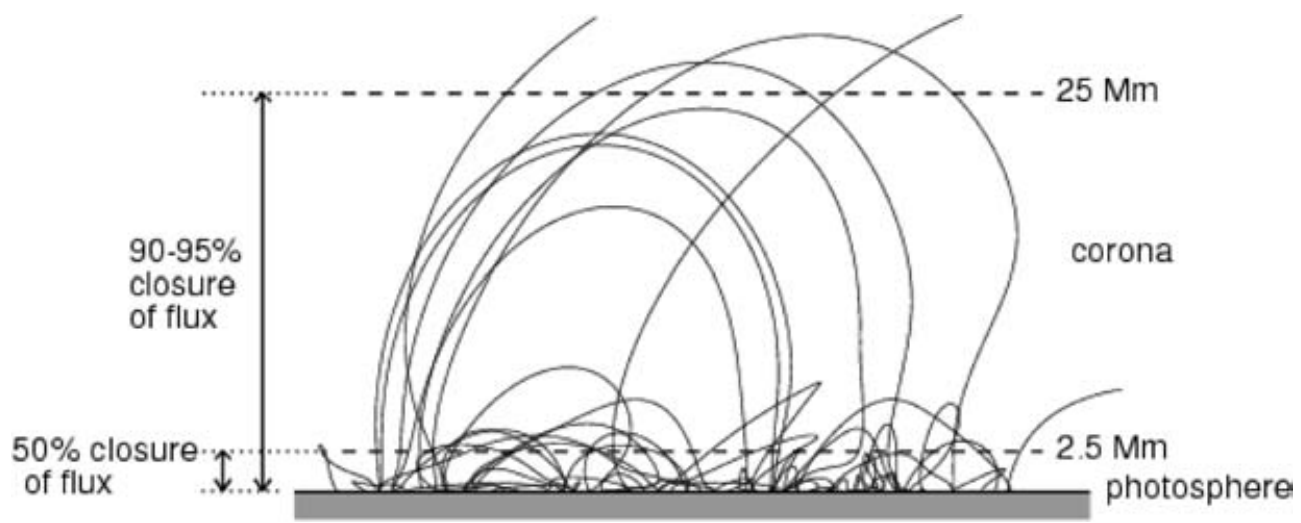

Figure 9. Coronal magnetic field lines computed from observed photospheric magnetograms (Close et al)

both in the current sheet and in the footpoints where downward propagating fast particles impact the lower atmosphere.

\subsection{Coronal Heating}

Magnetic reconnection is the likely mechanism for heating the solar corona and may operate in several ways. It may be driven directly by photospheric motions and produce $x$-ray bright points (Parnell et al, 1994) and transient brightenings (Shimizu, 2004). It may occur as "binary reconnection" when pairs of sources interact (Priest, Longcope and Titov, 2004). It may also take place by separator reconnection (Longcope, 2001) in complex magnetic fields or by braiding (Parker, 1972).

A new suggestion is that reconnection by "Coronal Tectonics" may be heating the corona (Priest, Heyvaerts and Title, 2002). The aim here is to determine the effect on coronal heating of the magnetic carpet (Schrijver et al, 1997), which is the name given to the spreading of flux into the corona from many small concentrated magnetic sources in the photosphere. Indeed, recently Close, Parnell and Priest (2004) have constructed the 
coronal magnetic field in the quiet Sun from observed magnetograms and have followed the photospheric sources in time and computed the time for all the magnetic field in the corona to reconnect - they found it is only 1.5 hours, so there is an incredible amount of reconnection continually taking place in the corona (Figure 9). Each coronal loop, however, reaches down to many sources in the photosphere and so the flux from each source is separated by separatrix surfaces. As the sources move, current sheets are formed on the separatrices and so the idea is that the corona is filled with myriads of current sheets, forming both on separatrices and separators and heating impulsively. Indeed, Priest and Longcope (2004) have just shown that separatrices tend to produce more heating than separators.

\section{Conclusions}

Solar physics is currently in a golden age, driven by amazing observations from space and ground. Magnetic theory is developing rapidly in an attempt to try and explain these puzzling observations, both of the photosphere (especially sunspots) and atmosphere (especially coronal loops and solar flares). Reconnection is thought to play a key role in solar flares and coronal heating. With future observations from a series of new missions (such as Solar B, Stereo, SDO and Orbiter) as well as the ground (ATST), it is clear that the current sense of vitality will continue - especially if there is a good supply of new young students eager to relish the opportunity.

\section{Acknowledgements}

I am most grateful to PPARC and ESA for financial support.

\section{References}

Carlsson, M. \& Stein, R. (2002), Astrophys. J. 572, 626-635

Close, R., Parnell, C.E. \& Priest, E.R. (2004), Astrophys. J. Letters, in press

Fletcher, L. (2004), these proceedings

Hornig, G. \& Priest, E.R. (2003), Phys. Plasmas 10, 2712-2721

Linton, M. \& Priest, E.R. (2003), Astophys. J. 595, 1259-1276

Longcope, D. (2001), Phys. Plasmas 8, 5277-5289

Parker, E.N. (1972), Astrophys. J. 174, 499-510

Parnell, C.E., Priest, E.R. \& Golub, L. (1994), Solar Phys. 151, 57-74

Pontin, D.I., Priest, E.R. \& Hornig, G. (2003), Solar Phys 212, 319-342

Priest, E.R. \& Forbes, T.G. (200), Magnetic Reconnection Cambridge University Press, Cambridge

Priest, E.R., Heyvaerts, J. \& Title, A. (2002), Astrophys. J. 576, 533-551

Priest, E.R., Hornig, G. \& Pontin, D.I. (2003), J. Geophys. Res. 108, SSH 6.1-6.8

Priest, E.R. \& Longcope, D. (2004), submitted

Priest, E.R., Longcope, D. \& Titov, V.S. (2004), Astrophys. J. in press

Rouppe vander Voort, L.H.M., Lofdahl, M.G., Kiselman, D. \& Scharmer, G.B. (2004), Astron. Astrophys. 414, 717-726

Scharmer, G.B., Gudiksen, B.V., Kiselman, D., Lofdahl, M.G. \& Rouppe van der Voort, L.H.M. (2002), Nature 420, 151

Schindler, K., Hesse, M. \& Birn, J. (1988), J. Geophys. Res. 93, 5547-5557

Shimizu, (2004), these proceedings

Schrijver, C.J., Title, A.M., van Ballegooijen, A.A., Hagenaar, H.J. \& Shine, R.A. (1997), Astrophys. J. 487, 424-436

Thomas, J.H., Weiss, N.O., Tobias, S.M. \& Brummel, N.H. (2002), Nature 420, 390

Weiss, N.O., Thomas, J.H., Tobias, S.M. \& Brummel, N.H. (2004), Astrophys. J. 600, 1073-1090 